\title{
Effect of error-contingent time-out on spaced responding in rats
}

\author{
ANDREW S. CZERWINSKI and ALBERT S. RODWAN \\ DePaul University, Chicago, Illinois 60614
}

\begin{abstract}
Under DRL 20- and 30-sec, rats' leverpresses with interresponse times shorter than the minimum interresponse time required for reinforcement produced a 15 - or 60 -sec time-out. The only systematic effect of the time-out procedure was to suppress responses with interresponse times shorter than 2 sec, suggesting that time-out functioned as stimulus feedback for a response. The effect of time-out was interpreted in terms of the dissimilarity between the operant and the consummatory response.
\end{abstract}

Under differential reinforcement of low rate (DRL) schedules, only responses separated by at least a minimum interresponse time (IRT), or t, are reinforced. Responses with IRTs shorter than $t$ merely restart the timing contingency.

When time-out (TO) followed responses with IRTs shorter than t, Kramer and Rilling (1969) found that pigeons' DRL performances were generally more accurate than during baseline sessions. Their TO procedure shifted IRT distributions in the direction of longer IRTs and suppressed responses with IRTs shorter than $2 \mathrm{sec}$. In a larger study, the present authors used a procedure similar to Kramer and Rilling's with a control group of rats. This group's data are of interest, since TO did not have the same effect on spaced responding as in Kramer and Rilling's study.

\section{METHOD}

\section{Subjects}

Three naive male Holtzman albino rats, 86 days old at the beginning of food deprivation, were maintained on an adjusted-80\% food-deprivation schedule (Davenport \& Goulet, 1964). The subjects were housed in individual cages, where they had continuous access to water.

\begin{abstract}
Apparatus
The lever in a standard Scientific Prototype Model A-110 operant chamber required $.15 \mathrm{~N}$ of force to activate its microswitch. The pellet dish was $3 \mathrm{~cm}$ to the left of the lever, and the chamber light was a $6-W$ bulb centered $10 \mathrm{~cm}$ above the lever. White noise and a fan used for ventilation masked extraneous sounds. Electromechanical counters, printers, and timers were situated in an adjacent room.
\end{abstract}

\section{Procedure}

After response shaping, $\mathrm{t}$ was incremented to $20 \mathrm{sec}$ during initial DRL training. Six phases were subsequently implemented. The schedule value $(\mathrm{t})$ was $20 \mathrm{sec}$ during Phases 1-3, and $30 \mathrm{sec}$

A version of this paper was presented at the meeting of the American Psychological Association, New Orleans, September, 1974. Thanks to John R. Platt for his comments on these data. Reprints may be obtained from Andrew S. Czerwinski, Department of Psychology, McMaster University, Hamilton, Ontario L8S 4K1, Canada. during Phases 4-6. Phases $1,3,4$, and 6 each consisted of 10 sessions under DRL $t$-sec. In Phases 2 and 5, the TO procedure was implemented during alternate sessions. Responses with IRTs shorter than $t$ produced TO during TO sessions, but had no scheduled consequences during no-TO sessions. Two TO durations, 15 and $60 \mathrm{sec}$, were counterbalanced. In both phases, Rat 1 was initially exposed to the longer duration, while Rats 2 and 3 were initially exposed to the shorter duration. During TO, the chamber light was turned off, and leverpresses had no scheduled consequences. For each subject, the implementation of each TO duration was continued on alternate sessions until the proportion of the total number of responses reinforced in a session did not vary by more than $15 \%$ across five consecutive TO sessions.

Throughout the experiment, each response with an IRT equal to or longer than $\mathrm{t}$ produced a standard $45-\mathrm{mg}$ Noyes food pellet. Interresponse times were measured from either the start of the session, the last reinforcement, the last response (Phases 1, 3,4 , and 6), or the last TO termination (Phases 2 and 5), whichever occurred most recently. Hence, TO periods were not defined as part of interresponse time. Sessions were terminated after $1 \mathrm{~h}$ of time-in, or functioning of the chamber light, and were conducted daily. The order of running of subjects was varied randomly across days.

\section{RESULTS AND DISCUSSION}

The subjects generally required more sessions to reach the criterion for stable performance for the first TO duration to which they were exposed under each DRL schedule (Table 1). The trends in Table 2 indicate that responding during TO decreased across time, suggesting that TO periods were discriminated.

Each subject's interresponse time per opportunity. (IRTs/OP; Anger, 1963) curves are shown in Figure 1, representing the last four sessions of Phases $1,3,4$, and 6 , and the last four sessions under each TO duration during Phases 2 and 5. This conditional probability statistic indicates the probability of a response at $\mathrm{t}+\mathrm{X} \mathrm{sec}$, given that the subject had waited $\mathrm{t}$ sec before responding. Following Kramer and Rilling (1969), no IRTs/OP values were computed where there were fewer than 20 opportunities.

The distributions indicate that unlike in Kramer and 
Table 1

Number of Sessions for Each TO Value Under Each DRL Schedule to Criterion for Stable Performance

\begin{tabular}{cccc}
\hline & \multicolumn{3}{c}{ Rats } \\
\cline { 2 - 4 } Schedule & No. 1 & No. 2 & No. 3 \\
\hline DRL 20-Sec & 5 & 8 & 11 \\
15-Sec TO & 5 & 9 & 5 \\
60-Sec TO & & & \\
DRL 30-Sec & 5 & 10 & 6 \\
15-Sec TO & 6 & 6 & 5 \\
60-Sec TO & &
\end{tabular}

Rilling's (1969) study, error-contingent TO did not decrease the probability of IRTs equal to or longer than $2 \mathrm{sec}$ but shorter than $\mathrm{tsec}$, and did not increase the probability of IRTs equal to or longer than $t$ sec. The only systematic effect of the TO procedure was to decrease bursting, or responses with IRTs shorter than $2 \mathrm{sec}$. Discontinuation of the TO procedure under each DRL schedule resulted in an increase in bursting. Since response bursts may be due to a lack of stimulus feedback for a response (cf. Kramer \& Rilling, 1970), TO periods may have served as stimulus feedback which decreased bursts. This interpretation should be viewed with caution, however, because IRTs measured from the previous TO termination were in effect response latencies (cf. Catania, 1970).

An alternate interpretation of the present results

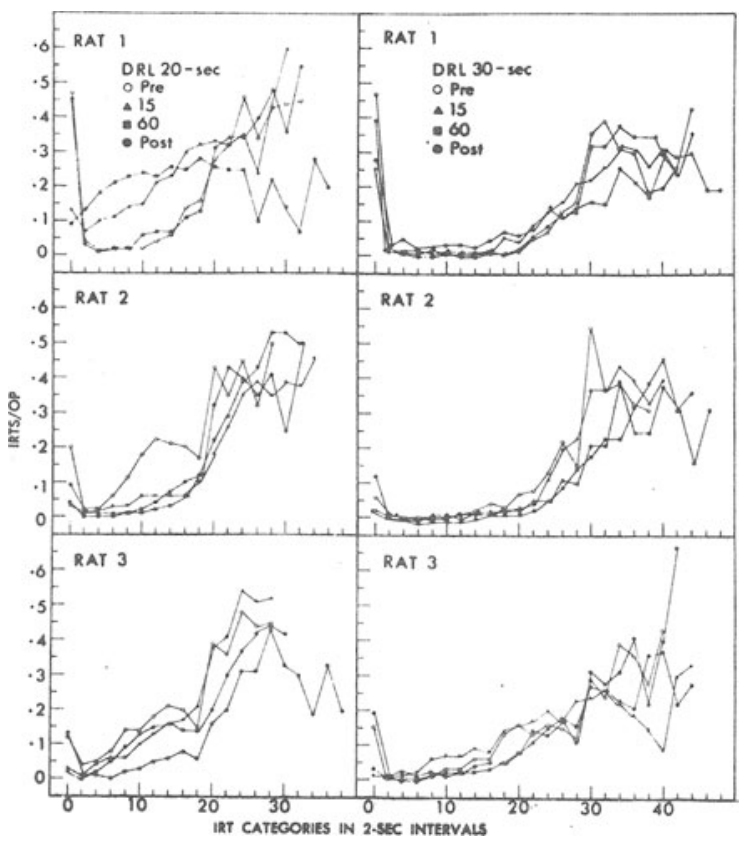

Figure 1. IRTs/OP values as a function of 2 -sec IRT categories. The numbers on the abscissa represent the lower limit of each IRT category. These data represent the Pre-TO phase (open circle), each of the two TO values (15-sec TO: triangle; 60-sec TO: square), and the post-TO phase (closed circle) under both DRL 20 - and 30 -sec.
Table 2

Responses/Minute During TO for the Last Four Sessions Combined for Each TO Value Under Each DRL Schedule

\begin{tabular}{cccc}
\hline & \multicolumn{3}{c}{ Rats } \\
\cline { 2 - 4 } Schedule & No. 1 & No. 2 & No. 3 \\
\hline DRL 20-Sec & & & \\
15-Sec TO & 4.09 & 0.83 & 1.31 \\
60-Sec TO & 2.22 & 0.44 & 0.74 \\
DRL 30-Sec & & & \\
15-Sec TO & 3.18 & 0.49 & 0.71 \\
60-Sec TO & 1.40 & 0.42 & 0.85 \\
\hline
\end{tabular}

involves the operant-consummatory response relationship. In DRL performance, the more similar the operant emitted by a species is to a consummatory response of that species, the more probable it is that the operant will be emitted (Hemmes, Note 1; Kramer \& Rodriguez, 1971). The importance of this relationship for the present experiment is suggested by Rat 1's data, which differ somewhat from the data for the other two subjects. Rat 1 met the criterion for terminating 15- and 60-sec TO sessions under both DRL schedules more quickly than did the other subjects (Table 1), and showed the highest response rate during TO (Table 2). In addition, this subject was more likely to emit response bursts than the other subjects, and generally displayed the poorest DRL performance, especially under DRL 20-sec phases (Figure 1).

The difference between Rat 1's data and that of the other subjects was apparently due to this subject's higher response rates. Such high response rates might be expected in light of the fact that the topography of this subject's leverpressing more clearly approximated rats' pellet-consumming response in the operant chamber. Casual observation indicated that, unlike the other subjects, Rat 1 consistently positioned the right side of the body flush against the front wall of the chamber while pressing the lever with the right hind paw. While in this position, this subject held the pellet dish with the front paws and generally kept the snout above it.

Thus, a major difference between this experiment and Kramer and Rilling's study entails expected response probabilities. Unlike in this experiment, the operant and consummatory response used by Kramer and Rilling were similar: both responses consisted of pecking. A possible conclusion is that for any species, error-contingent TO has a systematic suppressive effect and thus facilitates DRL performance only if the operant response resembles the consummatory response and thus response probability is high.

\section{REFERENCES}

Anger, D. The role of temporal discriminations in the reinforcement of Sidman avoidance behavior. Journal of the Experimental Analysis of Behavior, 1963, 6, 477-506.

Catania, A. C. Reinforcement schedules and psychophysical judgments: A study of some temporal properties of behavior. In W. N. Schoenfeld (Ed.), The theory of reinforcement schedules. New York: Appleton-Century-Crofts, 1970, Pp. 1-42. 
Davenport, D. G., \& Goulet, L. R. Motivational artifact in standard food-deprivation schedules. Journal of Comparative and Physiological Psychology, 1964, 57, 237-240.

Kramer, T. J., \& Rilling, M. Effects of time-out on spaced responding in pigeons. Journal of the Experimental Analysis of Behavior, $1969,12,283-288$

Kramer, T. J., \& Rilling, M. Differential reinforcement of low rates: A selective critique. Psychological Bulletin, 1970, 74, $225 \cdot 254$

Kramer, T. J., \& Rodriguez, .S. The effect of different operants on spaced responding. Psychonomic Science, 1971, 25, $177-178$.

\section{REFERENCE NOTE}

1. N. S. Hemmes, DRL efficiency depends upon the operant. Paper presented at the meeting of the Psychonomic Society, San Antonio, November, 1970.

(Received for publication December 18, 1974.) 